Pacific Journal of Mathematics

ISOMETRIC IMMERSIONS OF MANIFOLDS OF
NONNEGATIVE CONSTANT SECTIONAL CURVATURE 


\title{
ISOMETRIC IMMERSIONS OF MANIFOLDS OF NONNEGATIVE CONSTANT SECTIONAL CURVATURE
}

\author{
EDSEL STIEL
}

\begin{abstract}
Let $M^{d}$ denote a $C^{\infty}$ Riemannian manifold which is $d$ dimensional and complete. Our first result states that an isometric immersion of a flat $M^{d}$ into $(d+k)$-dimensional Euclidean space, $k<d$, is $n$-cylindrical if the relative nullity of the immersion has constant value $n$. This result was obtained by O'Neill with the additional hypothesis of vanishing relative curvature. We next consider the case in which $M^{d}$ and $\bar{M}^{d+k}, k<d$, are manifolds of the same constant positive sectional curvature. In this case we show that an isometric immersion of $M^{d}$ into $\bar{M}^{d+k}$ is totally geodesic if the relative curvature of the immersion is zero on a certain subset of $M^{d}$.
\end{abstract}

Let $M^{a}$ and $\bar{M}^{a+k}$ be $C^{\infty}$ Riemannian manifolds of the same constant sectional curvature $C, M^{a}$ being assumed complete and $k<d$. Let $\psi: M^{a} \rightarrow \bar{M}^{a+k}$ be an isometric immersion. The character of such immersions has been studied in [4] and [5] in terms of what Chern and Kuiper call the index of relative nullity of $\psi$ [2]. This function, $\nu$, assigns to each $m \in M$ the dimension of $\mathscr{N}(m)$, the subspace of vectors $x$ in the tangent space $M_{m}$ such that $T_{x}=0$. The linear difference operators $T_{x}$ act on $\bar{M}_{\psi(m)}$ and contain the same information as the classical second fundamental form operators $S_{z}$ where $z$ is a tangent vector to $\bar{M}$ orthogonal to $d \psi\left(M_{m}\right)$ [1]. In fact $T_{x}$ is characterized by its skew-symmetry and the equation $T_{x}(z)=d \psi\left(S_{z}(x)\right)$. Our first theorem concerns the case in which $M^{a}$ is flat and $\bar{M}^{a+k}=$ $R^{a+k}, d+k$ dimensional Euclidean space. It states that when $\nu$ is constant on $M^{d}$ the immersion $\psi$ is 'cylindrical'. We next investigate the corresponding situation for $C>0$.

We use essentially the notation in [4]. In particular we identify $M^{a}$ with $\psi\left(M^{a}\right)$ when it seems safe to do so. Let $N$ denote the bundle of normal $k$-frames of $M$ relative to $\psi$; that is

$$
\begin{aligned}
N= & \{(m, E) \mid m \in M \text { and } E \text { is a } k \text {-frame (orthonormal set of } k \\
& \text { vectors) of } \left.\bar{M}_{\psi(m)} \text { orthogonal to } d \psi\left(M_{m}\right)\right\} .
\end{aligned}
$$

The Riemannian connection of $\bar{M}^{a+k}$ induces a natural connection on $N$. The curvature form of this connection is called the relative curvature of $\psi$. We say that $\psi: M^{a} \rightarrow R^{a+k}$ is $n$-cylindrical provided

Received August 5, 1964. Part of this work was supported by the NSF while the author was a Research Assistant at UCLA. 
$M$ and $\psi$ can be expressed as Riemannian products $M^{a}=B^{a-n} \times R^{n}$ and $\psi=\bar{\psi} \times 1$ where $\bar{\psi}$ is an isometric immersion of $B^{a-n}$ in $R^{a+k-n}$ and 1 is the identity map of $R^{n}$. We can now state our first theorem precisely. This result was obtained by O'Neill as Theorem 2 of [4] but with an additional hypothesis, namely, the assumption of zero relative curvature. We shall use a similar assumption in our Theorem 3.

THeOREM 1. Let $M^{a}$ be a complete, flat, $C^{\infty}$ Riemannian manifold. An isometric immersion $\psi: M^{a} \rightarrow R^{a+k}$ is $n$-cylindrical if the relative nullity has constant value $n$.

We summarize some results applicable to an isometric immersion between two manifolds of constant curvature $C$. Let $\mathscr{N}^{\perp}(m)$ be the orthogonal complement of $\mathscr{N}(m)$ in $M_{m}$. From [5] we have: If $n$ denotes the minimum value of $\nu$, then $n \geqq d-k$ and $G$, the open subset of $M^{a}$ on which $\nu=n$, is foliated by complete totally geodesic subspaces (the leaves of $\mathscr{N}$ ) which are also totally geodesic relative to $\psi$. Also there exists for any $m \in G$ an $x \in \mathscr{N}^{\perp}(m)$ such that $T_{x}$ is injective on $\mathscr{N}^{\perp}(m)$. The two cases of interest to us are:

Case 1. $G=M^{d}$ (i.e., $\nu$ is constant), $\bar{M}^{d+k}=R^{d+k}(C=0)$ and $a=\infty$ (see below).

Case 2. $C>0$ and $0<a<\pi / 4 \sqrt{C}$.

The parameter $a$ appears in the following lemma. Let $\gamma:(-a, a) \rightarrow L$ be a unit speed geodesic in a leaf $L$ of $\mathscr{N}$ in $G$. Then there exists a frame field $E=\left(E_{1}, \cdots, E_{d+k}\right)$ on a neighborhood or $\gamma$ in $G$ such that:

1. The geodesic $\gamma$ is an integral curve of $E_{1}$;

2. Each integral curve of $E_{1}$ is a geodesic of $M$;

3. The vector fields $E_{1}, \cdots, E_{n}$ are contained in $\mathscr{N}, E_{n+1}, \cdots, E_{d}$ in $\mathscr{N}^{\perp}$, and $E_{d+1}, \cdots, E_{d+k}$ are contained in the orthogonal complement of $\psi\left(M_{m}\right)$ in $\bar{M}_{\psi(m)}$;

4. The frame $E$ is parallel on $\gamma$. The construction for this lemma is contained in Lemma 1 of [5], except we use the additional fact that the leaves of $\mathscr{N}$ are $R^{n}$ planes in Case 1 for $a=\infty$. We pull the connection form $\bar{\phi}$ of the frame bundle of $\bar{M}^{d+k}$ down to $G$ by way of the frame field $E$. Using the following index convention,

$$
\begin{array}{ll}
1 \leqq \alpha, b \leqq n ; & n+1 \leqq q, r, s \leqq d ; \\
1 \leqq i, j \leqq d ; & d+1 \leqq \alpha, \beta \leqq d+k,
\end{array}
$$




$$
\begin{array}{ll}
\phi_{i j}=\bar{\phi}_{i j} \circ d E & \text { (connection forms of } M), \\
\tau_{i \alpha}=\bar{\phi}_{i \alpha} \circ d E & \text { (Codazzi forms), } \\
\theta_{\alpha \beta}=\bar{\phi}_{\alpha \beta} \circ d E & \text { (normal connection forms). }
\end{array}
$$

A set of linear operators on $\mathscr{N}^{\perp}$ dependent on the frame field $E$ can be defined by

$$
P_{E_{\alpha}}\left(E_{s}\right)=\Sigma_{r} \phi_{r a}\left(E_{s}\right) E_{r} .
$$

From the second structural equation and the properties of the frame field $E$ one can show that the matrix $P(t)$ of $P_{\gamma^{\prime}(t)}$ satisfies the differential equation $P^{\prime}=-P^{2}-C I$ on $(-a, a)$ where $I$ denotes the $(d-n) \times(d-n)$ identity matrix. See Lemma 3 of [5]. Our proof of Theorem 1 hinges on the central result from [4] which states that if for all $m \in M^{a}$ and $x \in \mathscr{N}^{(m)}$ we have that $P_{x}=0$ then the immersion is n-cylindrical. Theorem 1 can now be easily proved with the help of the following lemma which is applicable in both Case 1 and Case 2.

Lemma 1. Let $m \in L$. If $x \in \mathscr{N}(m)$ and $y \in \mathscr{N}^{\perp}(m)$ then $T_{P_{x}(y)}=$ $T_{y} \circ P_{x}$ on $\mathscr{N}^{\perp}(m)$.

Proof. Since $L$ is complete there exists a geodesic $\gamma:(-a, a) \rightarrow L$ with $\gamma(0)=m$ and a frame field $E$ as defined above in a neighborhood of $\gamma$. From $T_{E_{i}}\left(E_{j}\right)=\Sigma_{\alpha} \tau_{\alpha j}\left(E_{i}\right) E_{\alpha}$ and the definition of $\mathscr{N}$ we get that $\tau_{\alpha a}=0$. Using this fact with the Codazzi equation for $\tau_{\alpha a}$ we have

$$
0=d \tau_{a \alpha}=-\Sigma_{i} \phi_{a i} \wedge \tau_{i \alpha}-\Sigma_{\beta} \tau_{a \beta} \wedge \theta_{\beta \alpha}=\Sigma_{q} \phi_{a q} \wedge \tau_{q \alpha} .
$$

This implies that

$$
\Sigma_{\alpha, q} \phi_{q a}\left(E_{s}\right) \tau_{\alpha q}\left(E_{r}\right) E_{\alpha}=\Sigma_{\alpha, q} \phi_{q a}\left(E_{r}\right) \tau_{\alpha q}\left(E_{s}\right) E_{\alpha}
$$

or that

$$
T_{E_{r}}\left(P_{E_{a}}\left(E_{s}\right)\right)=T_{E_{s}}\left(P_{E \alpha}\left(E_{r}\right)\right) .
$$

Hence for $x \in \mathscr{N}(m)$ and $y, z \in \mathscr{N}^{\perp}(m)$ we have

$$
T_{y}\left(P_{x}(z)\right)=T_{z}\left(P_{x}(y)\right)=T_{P_{x}(y)}(z),
$$

the last equality above following from the symmetry of the second fundamental form operators.

2. Proof of Theorem 1. We shall show that $P_{x}=0$ for $x \in \mathscr{N}(m)$, $m \in M^{d}$. We may assume $x$ is a unit vector and $\gamma$ is a unit speed com- 
plete geodesic of the leaf through $m$ with $\gamma^{\prime}(0)=x$. By a previous remark we may pick $y \in \mathscr{N}^{\perp}(m)$ such that $T_{y}$ is injective on $\mathscr{N}^{\perp}(m)$. Then $\mathscr{N}^{\perp}+T_{y}\left(\mathscr{N}^{\perp}\right)$ is invariant under both $T_{y}$ and $T_{P_{x}(y)}$. Hence the $2(d-n) \times 2(d-n)$ matrix of $T_{y} \mid\left(\mathscr{N}^{\perp}+T_{y}\left(\mathscr{N}^{\perp}\right)\right)$ can be represented by a $(d-n) \times(d-n)$ matrix $A$ in the upper right hand corner, $-A^{t}$ in the lower left hand corner and zeros elsewhere. If $B$ is the analogous block for $T_{P_{x}(y)}$ then $Q=-A B^{t}$ will be the matrix of $T_{y} \circ T_{P_{x}(y)} \mid \mathscr{N}^{\perp}$. The difference operators $T_{y}$ and $T_{P_{x}(y)}$ commute on $M_{m}$ since $M$ is flat and hence we have $A B^{t}=B A^{t}$. By Lemma 1, $P_{x}=T_{y}^{-1} \circ T_{P x(y)} \mid \mathscr{N}^{\perp}$ and hence $P(0)=\left(A^{-1}\right)^{t} B^{t}$. Let

$$
R=-A^{-1} Q\left(A^{-1}\right)^{t}=B^{t}\left(A^{-1}\right)^{t} .
$$

Since $Q$ is symmetric so is $R$ and therefore $P(0)$ has the same (real) eigenvalues as $R$. These eigenvalues satisfy $\lambda_{k}^{\prime}=-\lambda_{k}^{2}$ on the real line (since $P$ satisfies this equation by a result stated above) and hence each $\lambda_{k}=0$. Thus $R=0$ and this implies $P(0)=0$ which is the desired result.

3. Positive curvature case. For completeness we include Corollary 1 of [5] as

THeOREM 2. Let $M^{a}$ and $\bar{M}^{a+k}$ be $C^{\infty}$ manifolds with the same constant positive curvature $C, M^{a}$ being assumed complete. Let $\psi$ : $M^{a} \rightarrow \bar{M}^{a+k}$ be an isometric immersion with $2 k \leqq d$. Then $\psi$ is totally geodesic.

As above let $\mathrm{n}$ denote the minimum value of $\nu$ and let $G$ consist of the $m \in M^{d}$ for which $\nu(m)=n$.

THEOREM 3. Let $M^{a}$ and $\bar{M}^{a+k}$ be $C^{\infty}$ manifolds with the same constant positive curvature $C, M^{a}$ being assumed complete. Let $\psi$ : $M^{a} \rightarrow \bar{M}^{a+k}$ be an isometric immersion with $k<d$. Then $\psi$ is totally geodesic if the relative curvature of $\psi$ is zero on $G$.

Proof. The proof is by contradiction. If $\psi$ is not totally geodesic then $n<d$. Let $L$ be a leaf in $G$ and let $m \in L$. We first show that for any $x \in \mathscr{N}(m), P_{x}$ is a symmetric operator and is independent of the frame field used in its definition. Let $y \in \mathscr{N}^{\perp}(m)$ such that $T_{y}$ is injective on $\mathscr{N}^{\perp}$. Using a geodesic $\gamma:(-a, a) \rightarrow L$ with $\gamma^{\prime}(0)=x$ and Lemma 1 we have as in the proof of Theorem 1 that $P(0)=$ $\left(A^{-1}\right)^{t} B^{t}$. Since the relative curvature of $\psi$ is zero we get from the Ricci equation of the immersion that the Codazzi forms satisfy the relation $\Sigma_{i} \tau_{\alpha i} \wedge \tau_{i \beta}=0$. From this we conclude that $T_{y}$ and $T_{P x(y)}$ 
commute on $\left(d \psi\left(M_{m}\right)\right)^{\perp}$ or $A^{t} B=B^{t} A$. This equation implies that $P(0)$ is symmetric. From the first structural equation we have that

$$
\left[E_{r}, E_{s}\right]=\Sigma_{i}\left(\phi_{r i}\left(E_{s}\right)-\phi_{s i}\left(E_{r}\right)\right) E_{i}
$$

which together with the symmetry of $P_{x}$ implies $\left[E_{r}, E_{s}\right] \in \mathscr{N}^{\perp}$; thus $\mathscr{N}^{\perp}$ is integrable. For $x \in \mathscr{N}, P_{x}$ is actually a second fundamental form operator of the leaf through $\mathscr{N}^{\perp}$ and thus $P_{x}$ is independent of the choice of frame field used in its definition.

From the completeness of $L$ it follows that we can find a unit speed geodesic $\gamma$ in $L$ defined on the real line. Since $M$ is of constant positive curvature, $\gamma$ is a compact immersion and $P_{\gamma^{\prime}}$ is a periodic function on the real line. Let $\lambda$ be one of the $d-n$ real eigenvalue functions determined by the symmetric operator $P_{\gamma^{\prime}}$. We may assume $\lambda$ attains a maximum at $m=\gamma(0)$. Let $E$ be a frame field as above. Then $\lambda$ must satisfy $\lambda^{\prime}(0)=-\lambda^{2}(0)-C=0$ since $P$ satisfies $P^{\prime}=$ $-P^{2}-C I$ on an interval containing 0 . This implies $\lambda(0)$ is not real, which is the desired contradiction. Hence $n \geqq d$ or $\psi$ is totally geodesic on $M$.

As a Corollary we get a result of O'Neill's from [3]. Let $S^{d+1}(C)$ denote the sphere of curvature $C$.

Corollary 1. Let $M^{a}$ and $\bar{M}^{d+1}$ be $C^{\infty}$ manifolds with the same constant positive curvature $C, M^{d}$ being assumed complete. Then any isometric immersion $\psi: M^{d} \rightarrow \bar{M}^{d+1}$ is totally geodesic. In particular if $\bar{M}^{d+1}=S^{d+1}(C)$ then any such immersion is an imbedding onto a great sphere.

Proof. The vanishing of the relative curvature of $\psi$ is trivial in the hypersurface case. In case $\bar{M}^{d+1}=S^{d+1}(C)$ we have that $\psi(M)=$ $S^{d}(C) \subset S^{d+1}(C)$. Letting $\bar{S}^{d}(C)$ denote the universal covering manifold of $M^{d}$ and $\pi$ the natural projection, we have that $\psi \circ \pi$ is a local isometry onto $\psi(M)$. Hence $\psi \circ \pi$ and therefore $\psi$ is injective. Thus $\psi$ is an imbedding onto $S^{d}(C)$.

\section{REFERENCES}

1. W. Ambrose, The Cartan structural equations in classical Riemannian geometry, J. Indian Math. Soc. 24 (1960), 23-76.

2. S. S. Chern and N. H. Kuiper, Some theorems on the isometric imbedding of compact Riemann manifolds in Euclidean space, Ann. of Math. (2) 56 (1952), 422-430.

3. B. O'Neill, Isometric immersions which preserve curvature operators, Proc. Amer. Math. Soc. 13 (1962), 759-763.

4. - Isometric immersion of flat Riemannian manifolds in Euclidean space, Michigan Math. J. 9 (1962), 199-205.

5. B. O'Neill and E. Stiel, Isometric immersions of constant curvature manifolds, Michigan Math. J. 10 (1963), 335-339.

6. E. Stiel, Isometric Immersions of Riemannian Manifold, Doctoral Dissertation, University of California at Los Angeles, (1963).

California State College at Fullerton 



\title{
PACIFIC JOURNAL OF MATHEMATICS
}

\author{
EDITORS
}

\author{
H. SAmelson \\ Stanford University \\ Stanford, California \\ R. M. Blumenthal \\ University of Washington \\ Seattle, Washington 98105
}

\author{
J. DugundjI \\ University of Southern California \\ Los Angeles, California 90007
}

*Richard Arens

University of California

Los Angeles, California 90024

\section{ASSOCIATE EDITORS}
E. F. BECKENBACH
B. H. NeUmanN
F. WOLF
K. YoSIDA

\section{SUPPORTING INSTITUTIONS}

\author{
UNIVERSITY OF BRITISH COLUMBIA \\ CALIFORNIA INSTITUTE OF TECHNOLOGY \\ UNIVERSITY OF CALIFORNIA \\ MONTANA STATE UNIVERSITY \\ UNIVERSITY OF NEVADA \\ NEW MEXICO STATE UNIVERSITY \\ OREGON STATE UNIVERSITY \\ UNIVERSITY OF OREGON \\ OSAKA UNIVERSITY \\ UNIVERSITY OF SOUTHERN CALIFORNIA
}

\author{
STANFORD UNIVERSITY \\ UNIVERSITY OF TOKYO \\ UNIVERSITY OF UTAH \\ WASHINGTON STATE UNIVERSITY \\ UNIVERSITY OF WASHINGTON \\ * * * * \\ AMERICAN MATHEMATICAL SOCIETY \\ CALIFORNIA RESEARCH CORPORATION \\ SPACE TECHNOLOGY LABORATORIES \\ NAVAL ORDNANCE TEST STATION
}

Mathematical papers intended for publication in the Pacific Journal of Mathematics should by typewritten (double spaced). The first paragraph or two must be capable of being used separately as a synopsis of the entire paper. It should not contain references to the bibliography. No separate author's resumé is required. Manuscripts may be sent to any one of the four editors. All other communications to the editors should be addressed to the managing editor, Richard Arens, at the University of California, Los Angeles, California 90024.

50 reprints per author of each article are furnished free of charge; additional copies may be obtained at cost in multiples of 50 .

The Pacific Journal of Mathematics is published quarterly, in March, June, September, and December. Effective with Volume 13 the price per volume (4 numbers) is $\$ 18.00$; single issues, $\$ 5.00$. Special price for current issues to individual faculty members of supporting institutions and to individual members of the American Mathematical Society: $\$ 8.00$ per volume; single issues $\$ 2.50$. Back numbers are available.

Subscriptions, orders for back numbers, and changes of address should be sent to Pacific Journal of Mathematics, 103 Highland Boulevard, Berkeley 8, California.

Printed at Kokusai Bunken Insatsusha (International Academic Printing Co., Ltd.), No. 6, 2-chome, Fujimi-cho, Chiyoda-ku, Tokyo, Japan.

PUBLISHED BY PACIFIC JOURNAL OF MATHEMATICS, A NON-PROFIT CORPORATION

The Supporting Institutions listed above contribute to the cost of publication of this Journal, but they are not owners or publishers and have no responsibility for its content or policies.

* Basil Gordon, Acting Managing Editor until February 1, 1966. 


\section{Pacific Journal of Mathematics}

\section{Vol. 15, No. $4 \quad$ December, 1965}

Robert James Blattner, Group extension representations and the structure space ........... 1101

Glen Eugene Bredon, On the continuous image of a singular chain complex .............. 1115

David Hilding Carlson, On real eigenvalues of complex matrices .................... 1119

Hsin Chu, Fixed points in a transformation group ............................. 1131

Howard Benton Curtis, Jr., The uniformizing function for certain simply connected Riemann

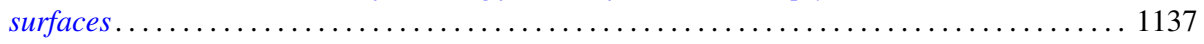

George Wesley Day, Free complete extensions of Boolean algebras................... 1145

Edward George Effros, The Borel space of von Neumann algebras on a separable Hilbert

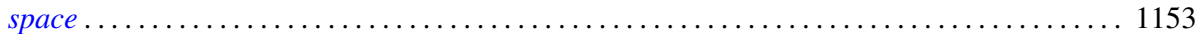

Michel Mendès France, $A$ set of nonnormal numbers ......................... 1165

Jack L. Goldberg, Polynomials orthogonal over a denumerable set ................ 1171

Frederick Paul Greenleaf, Norm decreasing homomorphisms of group algebras . . . . . . . . 1187

Fletcher Gross, The 2-length of a finite solvable group ........................ 1221

Kenneth Myron Hoffman and Arlan Bruce Ramsay, Algebras of bounded sequences ........ 1239

James Patrick Jans, Some aspects of torsion . . . . . . . . . . . . . . . . . . . . . . . 1249

Laura Ketchum Kodama, Boundary measures of analytic differentials and uniform

approximation on a Riemann surface ............................... 1261

Alan G. Konheim and Benjamin Weiss, Functions which operate on characteristic

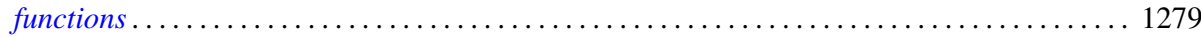

Ronald John Larsen, Almost invariant measures ............................ 1295

You-Feng Lin, Generalized character semigroups: The Schwarz decomposition ............ 1307

Justin Thomas Lloyd, Representations of lattice-ordered groups having a basis . . . . . . . . 1313

Thomas Graham McLaughlin, On relative coimmunity ....................... 1319

Mitsuru Nakai, $\Phi$-bounded harmonic functions and classification of Riemann surfaces ....... 1329

L. G. Novoa, On n-ordered sets and order completeness ..................... 1337

Fredos Papangelou, Some considerations on convergence in abelian lattice-groups . . . . . . . 1347

Frank Albert Raymond, Some remarks on the coefficients used in the theory of homology

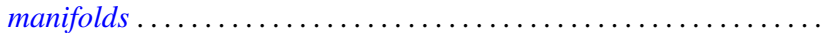

John R. Ringrose, On sub-algebras of a $C^{*}$-algebra .

Jack Max Robertson, Some topological properties of certain spaces of differentiable

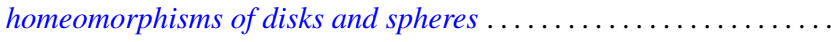

Zalman Rubinstein, Some results in the location of zeros of polynomials

Arthur Argyle Sagle, On simple algebras obtained from homogeneous general Lie triple systems. . . .

Hans Samelson, On small maps of manifolds ............................... 1401

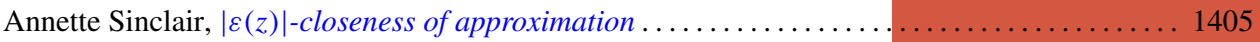

Edsel Ford Stiel, Isometric immersions of manifolds of nonnegative constant sectional curvature

Earl J. Taft, Invariant splitting in Jordan and alternative algebras ................. 1421

L. E. Ward, On a conjecture of R. J. Koch . . . . . . . . . . . . . . . . . . . . . . . . . . . 1429

Neil Marchand Wigley, Development of the mapping function at a corner . . . . . . . . . . 1435

Horace C. Wiser, Embedding a circle of trees in the plane ....................... 1463

Adil Mohamed Yaqub, Ring-logics and residue class rings . . . . . . . . . . . . . . . . 1465

John W. Lamperti and Patrick Colonel Suppes, Correction to: Chains of infinite order and their application to learning theory ........................................ 1471

Charles Vernon Coffman, Correction to: Non-linear differential equations on cones in Banach

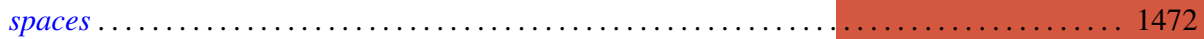

P. H. Doyle, III, Correction to: A sufficient condition that an $\operatorname{arc}$ in $S^{n}$ be cellular. . . . . . . . 1474

P. P. Saworotnow, Correction to: On continuity of multiplication in a complemented algebra 